\title{
QUANTIFICATION OF UNCERTAINTIES IN A 2D HYDRAULIC MODEL FOR THE DUTCH RIVER RHINE USING EXPERT OPINIONS
}

\author{
Jord J. Warmink ${ }^{1 *}$, Hanneke van der Klis ${ }^{2}$, Martijn J. Booij ${ }^{1}$ and Suzanne J.M.H Hulscher ${ }^{1}$ \\ ${ }^{1}$ Department of Civil Engineering, University of Twente, PO Box 217, 7500 AE Enschede, The Netherlands \\ ${ }^{2}$ Deltares, Delft, The Netherlands \\ e-mail: j.j.warmink@utwente.nl
}

Keywords: Uncertainty analysis, Uncertainty identification, Water management, Hydraulic modeling, Pedigree, Expert opinions.

\begin{abstract}
Hydraulic-morphological river models are applied to design and evaluate measures for purposes such as safety against flooding. These numerical models are all based on a deterministic approach. However, the modeling of river processes involves numerous uncertainties. The aim of this study is to identify the sources of uncertainty that induce the largest uncertainties in the model outcomes and quantify this uncertainty using expert opinions. Experts have been selected based on a Pedigree matrix. The selected experts are asked to list and quantify the most important uncertainty sources for two situations: (1) the computation of design water levels (DWL) and (2) the computation of the hydraulic effect of a change in the river bed. The experts stated that the sources of uncertainty are different for the computation of the DWL and effect studies. The experts agreed that for DWL, the upstream discharge and the roughness predictor for the main channel have the largest uncertainty. For effect studies, no clear dominant source could be identified. The quantification of the uncertainty sources showed a significant effect on the predicted water levels under design discharge conditions.
\end{abstract}

\section{INTRODUCTION}

Hydraulic-morphological river models are applied to design and evaluate measures for purposes such as safety against flooding. These numerical models are all based on a deterministic approach. However, the modeling of river processes involves numerous uncertainties, resulting in uncertain model results. Uncertainty is defined as any deviation from the unachievable ideal of complete determinism ${ }^{[1]}$. Uncertainty in models comprises (1) the difference between a model outcome and a measurement and (2) the possible variation around a computed value. According to Morgan and Henrion ${ }^{[2]}$, decision makers make less than optimal decisions without information on the uncertainties in outcomes. Knowledge of the type and magnitude of these uncertainties is crucial for a meaningful interpretation of the model results. Therefore, there is a need for structured analysis of the uncertainties in environmental management practice.

Most studies about uncertainty analysis of hydrodynamic models only take uncertainties in input and model parameters (e.g. ${ }^{[3]}$ and ${ }^{[4]}$ ) into account. However, uncertainty also resides in the model structure, computational characteristics, for example, discretizations and grid type, and in the model context. A structured approach is required to identify the uncertainties in all these locations in a model. Also, not all these uncertainties can be quantified. To get insight in the sources of uncertainty that influence the model outcomes, expert opinion elicitation is used as the method to identify the key uncertainties. The aim of this study is to identify the sources of uncertainty that induce the largest uncertainties in the model outcomes and quantify this uncertainty.

\section{METHOD}

\subsection{Study area}

In this study, the two-dimensional WAQUA model for the River Waal is used for the identification of sources of uncertainty. Shortly after the Rhine enters the Netherlands, it splits (by two subsequent bifurcations) into three main branches. The largest of these branches is the River Waal, which discharges about $2 / 3$ of the total Rhine discharge. Flood protection and navigation are the main issues in the river Waal region. Every 5 years the safety of the primary dikes is evaluated against a design discharge. This design discharge is based on the statistical analysis of historical discharge series. In 1999 a new policy for flood protection was adopted, the Room for the Rivers policy. This policy implies that instead of raising the dikes, if possible, other measures are taken to increase the discharge capacity of the river ${ }^{[5]}$.

The WAQUA model is used to compute the design water levels (DWL) based on the design discharge and to compute the effects of the Room for the River (RfR) measures. The results of this model are used for the decision, which leads to a set of measures in the river bed to ensure safety against flooding in the Netherlands. However, the uncertainty 
in the model outcomes are not explicitly considered in the decision making process.

The uncertainties in the outcomes of this model are a result of the uncertainties in all parts of the model, called the sources of uncertainty. Figure 1 shows a sketch of a general model. Uncertainties are present in the model input, parameters, computational characteristics and model structure ${ }^{[1]}$.

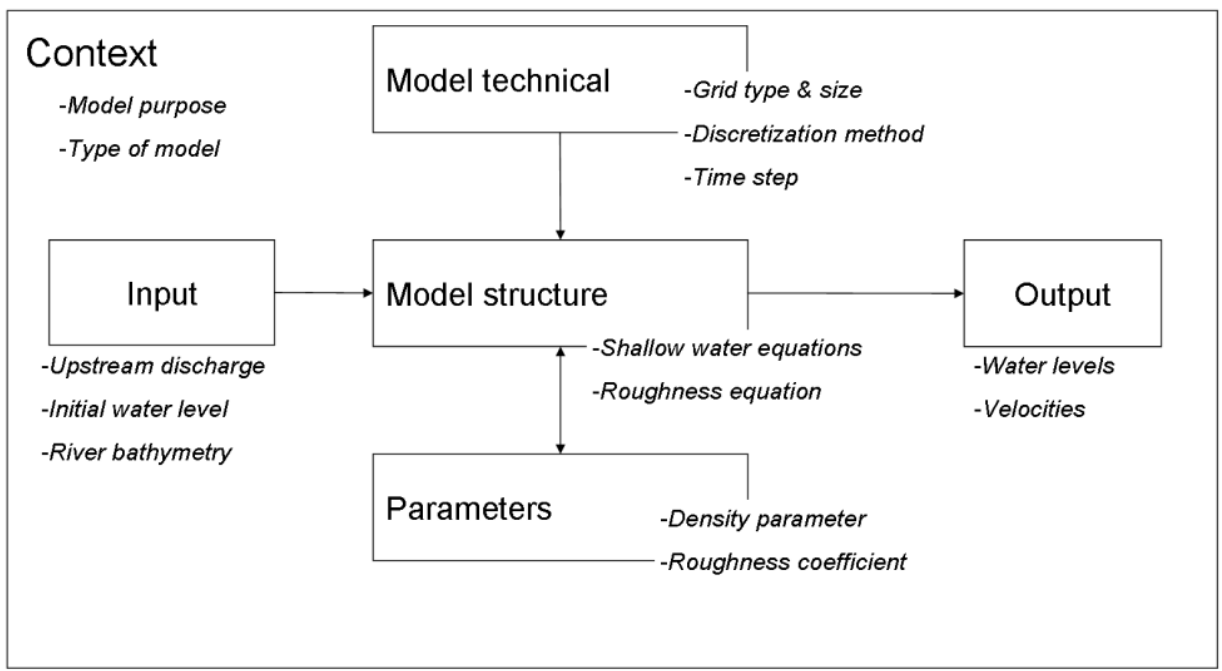

Figure 1: Locations of some major sources of uncertainty in a model that contribute to the model output uncertainty (based on Walker et al. ${ }^{[1]}$ )

\subsection{Expert selection}

At first 30 experts are asked for their experience with the WAQUA model. From these 30 experts, 16 are selected based on a Pedigree matrix ${ }^{[6]}$ with 4 criteria: 1) experience with code development, 2) experience with WAQUA projects, 3) number of years of 'hands-on' experience with WAQUA, and 4) number and type of publications about WAQUA. On each criterion a score between 0 and 4 has been given, based on the information given by the expert. Subsequently, the scores have been normalized and a weight factor is used for each criterion from 4 to 1 respectively. The 16 experts with the highest Pedigree scores have been invited for an interview. Interviews are held with 11 of these experts.

\subsection{Expert interviews}

The experts are asked to list the most important uncertainty sources. These are defined as the sources with the largest contribution to the model outcome uncertainties, which means that both the sources are highly uncertain and they have a large influence on the model outcomes. The experts are asked to consider the following two situations: (1) the computation of design water levels (DWL), based on a design discharge wave and (2) the computation of the hydraulic effect of a measure in the river bed, which is computed using a constant discharge as input. To compare the different experts, the experts are asked to comment on the sources of uncertainty on the same level of detail. Subsequently, the experts are asked to indicate the effect of a source of uncertainty on the computed water levels.

\section{RESULTS}

The experts stated that the sources of uncertainty are different for the computation of the DWL and effect studies. In case of effect studies, the experts agreed that the sources of uncertainty that do not change between the computation with and without a measure have little influence on the uncertainty in the computed effect. In case of DWL computations, the uncertainties are dominated by the sources that are not compensated during calibration.

\subsection{Uncertainties in design water levels}

The uncertainty in the DWL computations for different sources is shown in figure 2. Only the seven largest sources of uncertainty in the DWL are shown. Clearly, the upstream discharge, that is derived from the extrapolation of the historical discharge series, and the main channel roughness equation have a large uncertainty according to the experts. The other sources have an uncertainty in the order of magnitude of 5-10 cm. Besides the large values given for the order of magnitude of the uncertainty, also a large scatter is shown in the experts' opinions. 


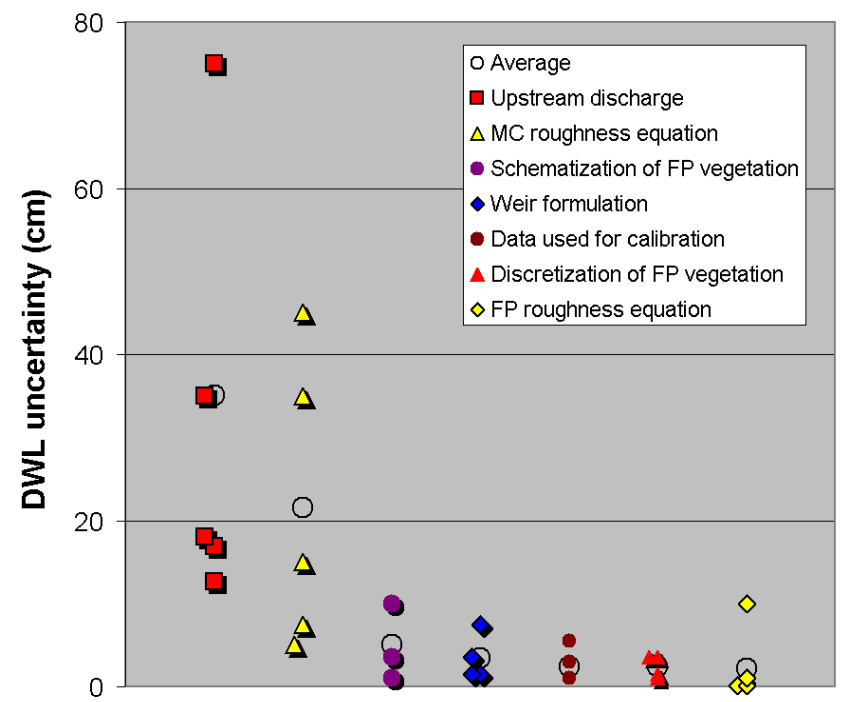

Figure 2: Uncertainty in the computed design water level (DWL), due to different uncertainty sources. The average uncertainty for each source (open circle) and the individual opinions of the experts are given for each uncertainty source. (MC is main channel; FP is floodplain).

\subsection{Uncertainties in effect studies}

Regarding the uncertainties in effect studies (figure 3), less experts were able to quantify the sources of uncertainty and the effect of uncertainty sources on model outcomes. This is mainly caused by the large dependency of the uncertainty on the location in the river bed. In general, the uncertainty in an effect study is important if it is different in the situation with a measure compared to the reference situation. If, for example, many weirs are changed, the uncertainty due to weirs has a relatively large influence. Due to this large dependency on (and interaction with) the local flow characteristics, uncertainty quantification becomes complicated. However for the Waal region several uncertainties are dominant. These uncertainties are the schematization of the vegetation, weirs and bathymetry in the floodplain area, the formulations of roughness due to vegetation and weirs, and the measurements of the floodplain bathymetry (figure $3)$.

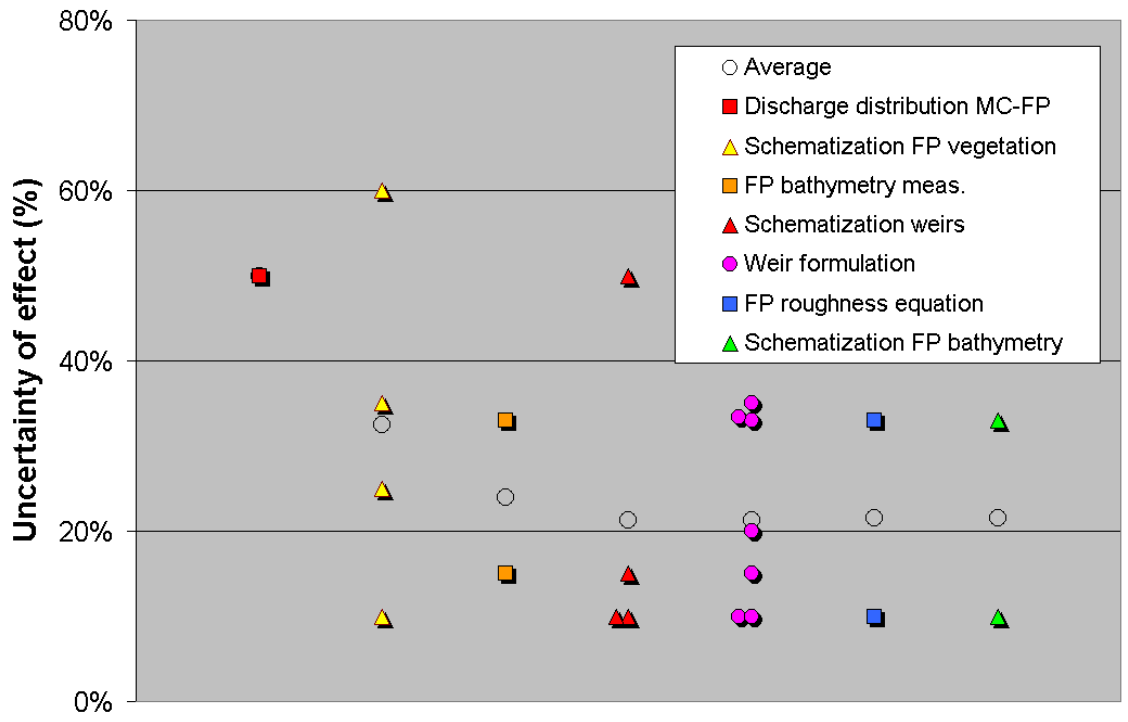

Figure 3: Sources of uncertainty for effect studies, expressed as a percentage of the computed effect. Also the mean (open circle) and the range of 1 standard deviation around the mean are given for each source of uncertainty.

\section{DISCUSSION}

According to the experts many uncertainties are reduced by calibration. This effect is taken into account in the estimation of the uncertainties. The uncertainties that are influenced by calibration are uncertainties in the measurement data, uncertainties in the discretization of this data onto a grid and the uncertainties in the computational parameters. 
This assumes that these parts of the model do not change between the situation used during calibration and the design conditions.

Other uncertainties that are not compensated for during calibration are valued by the experts to have a large effect on the uncertainty in the model outcomes. These uncertainties comprise the upstream discharge and the main channel roughness formulation. Furthermore, some experts state that the extrapolation to design conditions also introduces uncertainty in other parts of the model. This uncertainty mainly comes from the difference in water levels between the calibration conditions and the design conditions. This difference is especially large in the floodplain area and becomes apparent in the roughness formulations. Therefore, the formulation of the floodplain roughness formulation and the weir formulation is also stated as uncertain. For example, some experts question the validity of the weir formulation in case a large water level is present above the top of the weir.

The major difficulty in the determination of the main uncertainties is that all uncertainties are correlated. Therefore, many experts state that the discharge distribution between the floodplain and the main channel is of main importance. The ratio between both discharges expresses the ratio between the aggregated roughness of the main channel and the aggregated roughness of the floodplain area. In future studies, this characteristic should be taken into account in the calibration and validation of 2D hydrodynamic models. The uncertainty in this characteristic also expresses the uncertainty in the aggregated roughnesses.

The experts are also asked for the uncertainty sources for other models than the WAQUA model for the Waal. They stated that the dominant source of uncertainty is determined by the characteristics of the flow field and river geometry. For example, the uncertainty in the main channel roughness is much larger than the uncertainty in the vegetation roughness. However, for the IJssel River, the model outcome uncertainty is more dominated by uncertainty in vegetation roughness than for main channel roughness, because the floodplain areas are relatively large compared to the main channel.

\section{CONCLUSIONS}

The aim of this study is to identify the sources of uncertainty that induce the largest uncertainties in the model outcomes and quantify this uncertainty using expert opinions. The experts stated that the sources of uncertainty are different for the computation of the DWL and effect studies. In case of DWL computations, the uncertainties are dominated by the sources that do not change between the calibration and the prediction. The experts agreed that the imposed discharge and the empirical roughness equation for the main channel have a relatively large uncertainty. For effect studies, the floodplain topography, weir formulation and discretization of floodplain topography induces the largest uncertainty. Next to the large values given for the order of magnitude of the uncertainty, also a large scatter is shown in the experts' opinions. Finally, the effect of the uncertainty sources on the model outcomes showed that the uncertainty sources have a significant effect on the predicted water levels under design discharge conditions. Future research focuses on the quantification of these main sources of uncertainty.

\section{ACKNOWLEDGEMENTS}

This research is supported by the Technology Foundation STW, and the technology program of the Ministry of Economic Affairs. The authors thank all experts for their time and constructive input in the preparation stage and during the interviews.

\section{REFERENCES}

[1] Walker, W.E., Harremoës, P., Rotmans, J., Van der Sluijs, J.P., Van Asselt, M.B.A., Janssen, P.H.M. and Krayer von Kraus, M.P. (2003) "Defining uncertainty, a conceptual basis for uncertainty management in model-based decision support” Integrated Assessment, Vol. 4 (1), pp. 5-17

[2] Morgan, M.G., Henrion, M. (1990) "Uncertainty: a guide to dealing with uncertainty in quantitative risk and policy analysis" Cambridge University Press, New York

[3] Hall, J.W., Tarantola, S., Bates, P.D. and Horritt, M.S. (2005) "Distributed sensitivity analysis of flood inundation model calibration" Journal of Hydraulic Engineering, Vol. 131 (2), pp. 117-126

[4] Bates, P.D., Horritt, M.S., Aronica, G., Beven, K.J. (2004) "Bayesian updating of flood inundation likelihoods conditioned on flood extent data" Hydrological Processes, Vol. 18 (17), pp. 3347-3370

[5] Van Stokkom, H.T., Smits, A.J., Leuven, R.S. (2005) "Flood defence in the Netherlands: a new era a new approach" Water International, Vol. 30 (1), pp. 76-87

[6] Funtowicz, S.O. and Ravetz, J.R. (1990) “Uncertainty and Quality in Science for Policy”, Kluwer, Dordrecht. ISBN 0-7923-0799-2 